\title{
Effects of Biphalin on Corneal Epithelial Wound Healing ${ }^{\dagger}$
}

\author{
Erdost Yıldız 1,* , Özgün Melike Gedar Totuk ${ }^{2}$, Adriano Mollica ${ }^{3}$, Kerem Kabadayı ${ }^{2}$ \\ and Afsun Şahin ${ }^{1}$
}

1 Koç University Research Center for Translational Medicine, Koç University, 34010 Istanbul, Turkey; afsahin@ku.edu.tr

2 School of Medicine, Bahçeşehir University, 34734 Istanbul, Turkey; melikegedar@me.com (Ö.M.G.T.); keremkabadayi@outlook.com.tr (K.K.)

3 Department of Pharmacy, Università degli Studi G. d'Annunzio Chieti e Pescara, 66100 Chieti, Italy; a.mollica@unich.it

* Correspondence: erdostyildiz17@ku.edu.tr

+ Presented at the 2nd International Cell Death Research Congress, Izmir, Turkey, 1-4 November 2018.

Published: 5 December 2018

\begin{abstract}
After physical or surgical damage of corneal epithelium, most of analgesic drugs, like nonselective opioid agonists and non-steroid anti-inflammatory drugs, cannot be used because of their negative effects on wound healing process. Biphalin is selective $\mu$ and $\Delta$ opioid receptor agonist which has proven analgesic effects on rodents. Our purpose of study is finding effects of biphalin on wound healing of corneal epithelium. We used primary culture of human corneal epithelial cells (HCECs) for examining effects of biphalin on wound healing. Firstly, we measured toxicity of Biphalin in various concentrations with MTT assay and we showed biphalin has no toxic effects on HCECs in lower concentrations than $100 \mu \mathrm{M}$ in various incubation times. After MTT assay, we administered $1 \mu \mathrm{M}$ and $10 \mu \mathrm{M}$ biphalin at in vitro scratch assay of HCECs, biphalin increased wound closure process significantly at $1 \mu \mathrm{M}$ concentration $(p<0.05)$. Then we tested effects of biphalin on cell migration and proliferation separately. Bifalin increased migration of HCECs significantly $(p<0.01)$ at transwell migration assay. But we did not observe any significant difference between groups in Ki67 proliferation assay. In all these experiments, we also used naloxone to inhibiting effects of biphalin. In biphalin plus naloxone groups, effects of biphalin decrease partially. Our study results suggest, biphalin has positive effects on epithelial wound healing via opioid receptors. This effect because of increased migration of HCECs under influence of biphalin. With these findings, we propose biphalin as a new analgesic agent for post-surgical and post-traumatic care of corneal epithelial wounds.
\end{abstract}

Keywords: analgesics; opioids; corneal injury; corneal epithelium; cell culture; opioid receptors

\section{Introduction}

Opioids have three receptor classes from the heterotrimeric transmembrane G-protein-bound opioid receptor class (GCPR): Delta-opioid (DOR), kappa-opioid (KOR) and mu-opioid (MOR). Opioids show their cytoprotection, analgesia, neuroendocrine regulation, immunomodulation, and behavior modification effects with these receptors. Opioid receptors have shown in various layers of the eye of humans, rabbits and rats. They have roles on regulation of iris, accommodation power, aqueous humor dynamics, corneal wound healing, retinal development and reduction of intraocular pressure [1]. But we still do not know which pharmacological mechanisms are under these effects. Both DOR and MOR are defined in the corneas of animals. The effects of these two receptors on wound healing and migration were shown in separate groups by DOR and MOR knockout mice [2,3]. 
Biphalin (PubChem CID: 5487663) firstly synthesized by Lipkowski et al. [4] in 1982 and then resynthesized by Mollica et al. [5]. Biphalin is a dimeric nonspecific enkephalin analog, resulting in a complete analgesic response in the central nervous system by activation of the DORs and MORs. Biphalin has been shown to be one of the most potent, peptide-based, opioid analgesics. The analgesic power of biphalin when applied into the cerebral ventricles was found to be seven times higher than etorphine and three times higher than morphine [6]. According to our literature search, the role of synthetic opioid peptides on corneal epithelial cells has not been investigated in vitro. Our study aims to evaluate the effects of biphalin on human corneal epithelial cells, especially on wound healing, cell migration and proliferation.

\section{Materials and Methods}

In Vitro Live Migration Assay: Human immortalized corneal epithelial cells (HCECs) were grown to confluence on 12-well culture dishes. On reaching confluence, cells were rinsed with PBS and exposed to a differentiation medium consisting of Dulbecco modified Eagle medium (DMEM) with $10 \%$ fetal bovine serum (FBS), penicillin, and streptomycin for one day. Two perpendicular linear scratches were made using a sterile $200-\mu \mathrm{L}$ pipette tip and the wells were washed with PBS. Immediately after the scratch, all groups incubated in keratinocyte serum-free medium (KSFM; Gibco, Waltham, MA, USA). Biphalin $(1 \mu \mathrm{M}$ and $10 \mu \mathrm{M})$ or Biphalin plus Naloxone solution $(1 \mu \mathrm{M}$ and $10 \mu \mathrm{M}$ ) or their vehicle (PBS) were added to the cultures. The scratch area was captured hourly for $24 \mathrm{~h}$ using a live cell microscopy (DMi 8; Leica, Wetzlar, Germany) and photographed. The remaining wound area (RWA) was measured using Image software (NIH, Bethesda, MD, USA).

Transwell Migration Assay: Immediately after wounding process as mentioned above, HCECs were trypsinized, washed, and plated $\left(2.5 \times 10^{5}\right.$ cells per insert $)$ in $8.0-\mu \mathrm{m}$ pore size transwell inserts in KSFM. The lower compartment was filled with DMEM with $10 \%$ FBS. Experiment groups are treated as mentioned above. After $24 \mathrm{~h}$; the cells on the upper side of the insert were removed by scraping and the cells that had migrated through were fixed on the lower side of the membrane with $4 \%$ paraformaldehyde, then stained with hematoxylin-eosin dye and quantified by counting the number of cells in 10 separate fields. The data were expressed as the number of migrated cells per micrograph field for each sample well.

Ki67 Proliferation Assay: HCECs were plated in equal numbers in 24-well culture dishes. After reaching confluence, cells were rinsed with PBS and exposed to DMEM with 10\% FBS. Two perpendicular linear scratches were made using a sterile $200-\mu \mathrm{L}$ pipette tip and the wells were washed with PBS and incubated with KSFM without supplements. Experiment groups are treated as mentioned above. The cells were incubated for $6 \mathrm{~h}$ in $37^{\circ} \mathrm{C}$. After treatment, cells grown on 24-well culture dishes were fixed in $4 \%$ paraformaldehyde. After washes with PBS, the cells were incubated with $0.1 \%$ TritonX-100 in PBS. Then cells were incubated with Superblock (Thermo Scientific, Waltham, MA, USA). The cells then were incubated overnight at $4{ }^{\circ} \mathrm{C}$ with the rabbit anti-Ki67 primary antibody (Abcam, Cambridge, MA, USA) at optimal dilutions. After washes with PBS, cells were incubated with the FITC-conjugated secondary antibody for $90 \mathrm{~min}$ at $37^{\circ} \mathrm{C}$, then washed, counterstained with 406-diamidino-2-phenylindole (DAPI) and mounted. Negative controls were stained in a similar fashion.

Cytotoxicity Assay: HCECs were treated with different concentrations of Biphalin (from $1 \mathrm{pM}$ to $100 \mu \mathrm{M}$ ) in 96-well culture dishes for $24 \mathrm{~h}$. After incubation, color of MTT tetrazole salt was measured with a spectrophotometer at the wavelength of $570 \mathrm{~nm}$.

Statistical Analysis: Each experiment was replicated at least two times. Values were displayed as mean $\pm \mathrm{SD}$. Comparisons between groups were analyzed by two-way ANOVA. Results were considered statistically significant for $p<0.05$.

\section{Results}

At MTT toxicity assay, biphalin has no cytotoxic effects at doses lower than $10 \mu \mathrm{M}$ in the 24-h drug incubation. After MTT assay, we applied biphalin and biphalin plus naloxone in two different 
non-toxic doses, $1 \mu \mathrm{M}$ and $10 \mu \mathrm{M}$, at in-vitro wound healing model. We observed a statistically significant increase $(p<0.05)$ in relative wound area closure in Biphalin treated cells at $1 \mu \mathrm{M}$ concentration compared to the vehicle group (Figure 1). However, there is no statistically significant difference between $10 \mu \mathrm{M}$ biphalin concentration, both concentrations of biphalin plus naloxone and vehicle group (Figure 1a).

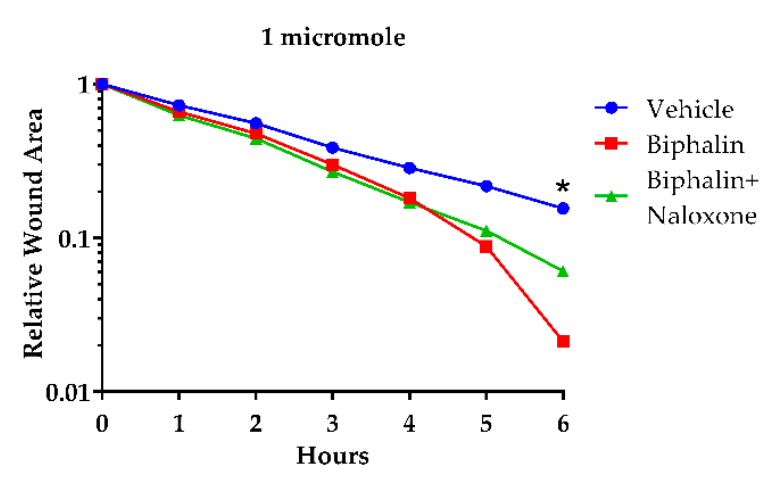

(a)

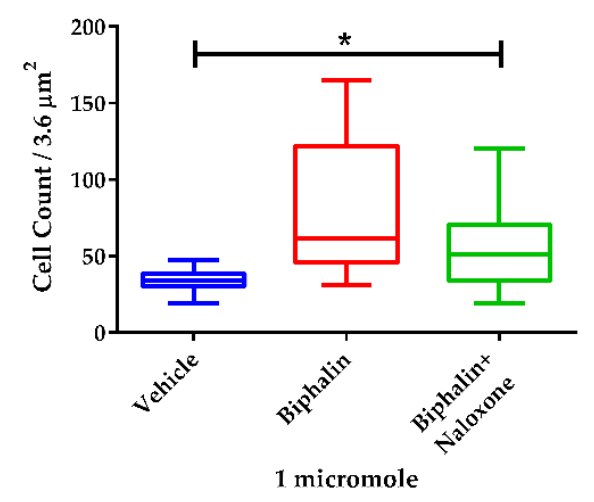

(b)

Figure 1. (a) Effect of biphalin and biphalin plus naloxone at 1 and $10 \mu \mathrm{M}$ concentrations on wound healing rate (RWA) in in vitro wound healing model. At $1 \mu \mathrm{M}$ concentration, biphalin treatment $(0.21$ \pm 0.23 ) has statically significant positive effect on HCECs compared to vehicle $(0.155 \pm 0.169)$ and biphalin plus naloxone $(0.061 \pm 0.063)$ groups $(n=8, * p<0.05)$. (b) Effects of biphalin and biphalin plus naloxone on the movement of corneal epithelial cells in the transwell migration assay. Both graphs show the average number of migrated cells in ten different micrograph area $\left(3.6 \mu \mathrm{M}^{2}\right)$ in two independent experiments. At $1 \mu \mathrm{M}$ concentration, biphalin treatment $(82.35 \pm 42.96)$ has statically significant positive effect on HCECs compared to vehicle $(34.35 \pm 7.08)$ and biphalin plus naloxone $(52.75 \pm 23.76)$ groups $(n=20, * p<0.05)$.

We observed, a statistically significant increase $(p<0.05)$ at the number of cells passing through the membrane in the $1 \mu \mathrm{M}$ concentration of biphalin at transwell migration assay. At the $10 \mu \mathrm{M}$ concentration of biphalin, although increased number of cells passing through the membrane, we could not show statistically significant difference between this group and control. In both concentrations of biphalin plus naloxone groups were not significantly different from vehicle groups. (Figure 1b) Lastly at Ki67 proliferation assay, we did not observe any statistically significant differences between experimental groups.

\section{Discussion}

We demonstrated the effect of synthetic opioid receptor agonists on corneal epithelium for the first time. Biphalin, which acts mainly via mu and delta opioid receptors, increases cell migration at wound area and accelerates wound closure without effecting cell proliferation. Partial inhibition of the wound healing effect of biphalin with naloxone shows that its effect on wound healing and migration is because of mu and delta opioid receptors (MOR and DOR).

Due to the retardant effects of wound healing, the use of non-selective opioids and non-steroidal anti-inflammatory drugs are unlikely to be used at post-trauma or post-operative patients. Previously, analgesic and antinociceptive effects of biphalin have been demonstrated in many studies. In our study, we showed the effect of biphalin on the cellular kinetics and cell cycle of corneal epithelium. We have demonstrated that it does not affect proliferation while accelerating wound healing and cell migration. We believe that the use of biphalin may be a new treatment alternative 
not only for corneal traumas but also for other neuropathic pain syndromes like herpetic or diabetic neuropathy, which are affecting the ocular surface.

Acknowledgments: The authors gratefully acknowledge use of the services and facilities of the Koç University Research Center for Translational Medicine (KUTTAM), funded by the Republic of Turkey Ministry of Development. The content is solely the responsibility of the authors and does not necessarily represent the official views of the Ministry of Development.

\section{References}

1. Husain, S.; Potter, D.E. The opioidergic system: potential roles and therapeutic indications in the eye. J. Ocul. Pharmacol. Ther. 2008, 24, 117-140, doi:10.1089/jop.2007.0112.

2. Bigliardi, P.L.; Neumann, C.; Teo, Y.L.; Pant, A.; Bigliardi-Qi, M. Activation of the delta-opioid receptor promotes cutaneous wound healing by affecting keratinocyte intercellular adhesion and migration. Br. J. Pharmacol. 2015, 172, 501-514, doi:10.1111/bph.12687.

3. Wang, Y.; Gupta, M.; Poonawala, T.; Farooqui, M.; Li, Y.; Peng, F.; Rao, S.; Ansonoff, M.; Pintar, J.E.; Gupta, K. Opioids and opioid receptors orchestrate wound repair. Transl. Res. 2017, 185, 13-23, doi:10.1016/j.trsl.2017.05.003.

4. Lipkowski, A.W.; Konecka, A.M.; Sroczynska, I. Double-enkephalins--synthesis, activity on guinea-pig ileum, and analgesic effect. Peptides 1982, 3, 697-700.

5. Mollica, A.; Davis, P.; Ma, S.W.; Lai, J.; Porreca, F.; Hruby, V.J. Synthesis and biological evaluation of new biphalin analogues with non-hydrazine linkers. Bioorg. Med. Chem. Lett. 2005, 15, 2471-2475, doi:10.1016/j.bmcl.2005.03.067.

6. Horan, P.J.; Mattia, A.; Bilsky, E.J.; Weber, S.; Davis, T.P.; Yamamura, H.I.; Malatynska, E.; Appleyard, S.M.; Slaninova, J.; Misicka, A.; et al. Antinociceptive profile of biphalin, a dimeric enkephalin analog. J. Pharmacol. Exp. Ther. 1993, 265, 1446-1454.

(C) 2018 by the authors. Licensee MDPI, Basel, Switzerland. This article is an open access article distributed under the terms and conditions of the Creative Commons Attribution (CC BY) license (http://creativecommons.org/licenses/by/4.0/). 